\title{
Влияние разогрева носителей заряда собственным стимулированным пикосекундным излучением в GaAs на линейное возрастание на фронте и длительность спектральной компоненты этого излучения
}

\author{
(C) Н.Н. Агеева, И.Л. Броневой , Д.Н. Забегаев, А.Н. Кривоносов \\ Институт радиотехники и электроники им. В.А. Котельникова Российской академии наук, \\ 125009 Москва, Россия \\ ฯE-mail: bil@cplire.ru
}

Поступила в Редакцию 17 декабря 2020 г.

В окончательной редакции 17 января 2021 г.

Принята к публикации 25 января 2021 г.

Во время мощной оптической пикосекундной накачки тонкого слоя GaAs, входящего в состав гетероструктуры $\mathrm{Al}_{x} \mathrm{Ga}_{1-x} \mathrm{As}-\mathrm{GaAs}-\mathrm{Al}_{x} \mathrm{Ga}_{1-x} \mathrm{As}$, в нем возникает интенсивное стимулированное пикосекундное излучение. При анализе измеренных в реальном времени импульсов спектральных компонент излучения установлены коэффициенты экспоненциального, потом линейного усиления компонент на фронте. При этом обнаружено влияние на фронт компонент, оказываемое нагревом носителей заряда излучением. Получена зависимость длительности компоненты (FWHM) от характерных времен возрастания на фронте и релаксации (тоже замедленной нагревом носителей излучением) на спаде компоненты.

Ключевые слова: стимулированное пикосекундное излучение, арсенид галлия, спектральные компоненты излучения, коэффициент усиления, характерное время возрастания излучения, характерное время релаксации излучения, разогрев носителей заряда, линейное усиление.

DOI: 10.21883/FTP.2021.05.50832.9577

\section{1. Введение}

В начале мощной пикосекундной оптической накачки тонкого слоя GaAs, входящего в состав гетероструктуры $\mathrm{Al}_{x} \mathrm{Ga}_{1-x} \mathrm{As}-\mathrm{GaAs}-\mathrm{Al}_{x} \mathrm{Ga}_{1-x} \mathrm{As}$, в нем возникает стимулированное пикосекундное излучение. Далее, для краткости, оно называется $s$-излучение. Спектр $s$-излучения представляет собой световой континуум. Отдельные аспекты спектра, как интегрального по времени, так и мгновенного, исследовались в наших предыдущих работах. Спектры $s$-излучения неоднократно приводились в наших статьях: (а) интегральные по времени спектры - в [1] и др.; (б) мгновенные спектры в [2] и др. Максимальная интенсивность интегрального по спектру $s$-излучения достигала при соответствующей накачке $1 \Gamma \mathrm{BT} / \mathrm{cm}^{2}$. В прошедшее время нами в большей степени исследовалась динамика $s$-излучения, интегрального по спектру, результаты кратко перечислены в [3]. Исследование спектральных компонент $s$-излучения пока ограничивалось измерениями длительности, момента достижения максимума, характерного времени релаксации компонент, вынужденного комбинационного рассеяния с их участием, их интерференции, автомодуляции. Для краткости импульс спектральной компоненты $s$-излучения будем называть $s$-компонента. Предлагаемая работа пополняет информацию о динамике $s$-компонент, выявляя параметры сначала экспоненциального, затем линейного возрастания на фронте, а также влияние на последнее разогрева носителей заряда $s$-излучением. Отметим, что влияние разогрева носителей излучением имеет, согласно [4], форму отрицательной обратной связи (ООС), тормозящей возрас- тание интенсивности $s$-компоненты и инверсии заселенности. Линейное усиление стимулированного излучения пока, насколько нам известно, исследовалось мало и в основном теоретически. В работе эмпирически получено простое выражение, связывающее длительность $s$-компоненты (FWHM) с двумя характерными временами: возрастания на фронте и релаксации на спаде $s$-компоненты. При этом характерное время релаксации $s$-компоненты в свою очередь определяется характерным временем остывания носителей и пропорционально ему [3] $]^{1}$.

\section{2. Экспоненциальное возрастание, релаксация и их связь с длительностью $s$-компоненты}

В настоящей работе мы опираемся на измерения в реальном времени огибающих $s$-компонент. Техника таких измерений достаточно подробно описывалась нами в предыдущих работах, например в [5], и мы не будем это повторять. Результат измерения в реальном времени огибающей импульса накачки и огибающей $s$-компоненты, представленные в графическом виде, будем называть хронограммами и обозначать $I_{\mathrm{ex}}(t)$ и $I_{s-m}(t)$. Излучение генерировалось в слое $\mathrm{GaAs}$ толщиной 1.5 мкм

\footnotetext{
1 Заметим, что ссылки на наши предыдущие работы приводятся вынужденно, а не для саморекламы. Заменить их ссылками на работы других авторов не удается, возможно, вследствие специфичности спектрофотохронометрического комплекса, на котором велись исследования. Перечень наших работ, посвященных исследованию $s$-излучения, приведен на сайте ИРЭ http://www.cplire.ru/rus/bil/index.html.
} 
упомянутой гетероструктуры при его накачке световым импульсом с энергией фотона $\hbar \omega_{\mathrm{ex}} \approx 1.512$ эВ, длительностью (FWHM) $T_{\mathrm{ex}} \approx 10$ пс, диаметром луча в местоположении образца (FWHM) $D=0.5$ мм. Площадь образца $-4 \times 4 \mathrm{MM}^{2}$. Частота генерации импульсов накачки составляла 1.4 Гц. Слои $\mathrm{Al}_{x} \mathrm{Ga}_{1-x} \mathrm{As}$ толщиной $\sim 1.3$ мкм прозрачны для света с $\hbar \omega<1.7$ эВ, используемого в экспериментах. На поверхности гетероструктуры (исключая поверхность торцов) было нанесено антиотражающее покрытие. Благодаря антиотражающему покрытию отражение света накачки от поверхности образца не превышало $2 \%$. Измерялась часть $s$-излучения GaAs, выходившая из накачанной области слоя и распространявшаяся внутри телесного угла $1.2 \cdot 10^{-2}$ стерадиан с осью, ортогональной поверхности гетероструктуры. Поясним это. Большая часть $s$-изучения, генерируемого в активной среде, выходит из нее в направлении вдоль слоев гетероструктуры. Остальная часть $s$-излучения выходит под разными углами из-за его упругого рассеяния на оптических неоднородностях, всегда имеющихся в реальных средах, (подробнее об этом см. в [5]). Представление о распределении по углам энергии $s$-излучения дает диаграмма направленности в [6]. $S$-излучение, выходящее из активной среды ортогонально гетерограницам, не искажается поглощением в пассивной среде. Поэтому хронограммы относительной интенсивности его $s$-компонент $I_{s-m}(t)$ были выбраны для определения режимов генерации и соответственно усиления интенсивности $s$-компонент со временем. То, что $s$-излучение (и выходящее из торца образца, и вытекающее через поверхность гетероструктуры из-за ее несовершенства) одно и то же - стимулированное, доказано в наших предыдущих исследованиях (доказательства суммированы в [5]). Рис. 1 иллюстрирует хронограммы света накачки и избранных $s$-компонент.

Все измеренные $s$-компоненты с энергией фотона в диапазоне $\hbar \omega=1.381-1.434$ эВ сначала возрастали со временем экспоненциально, приблизительно с того момента, обозначенного $t \approx 0$, с которого накачка начинала интенсивно возрастать (рис. 1). Это подтверждает касающийся хронограммы $s$-компоненты на ее начальном участке график 2 экспоненты на рис. 2.

Указанный спектральный диапазон $s$-компонент представляется естественным, поскольку стимулированное излучение возникает в $\mathrm{GaAs}$ обычно при плотности неравновесных носителей $n=p \approx(1.3-1.6) \cdot 10^{18} \mathrm{~cm}^{-3}$, при которой ширина запрещенной зоны $E_{g}$, перенормированной из-за кулоновского взаимодействия носителей, должна быть уже не более энергии фотона $\hbar \omega_{e} \approx 1.38$ эВ длинноволнового края спектра исследуемого излучения [7]. Указанное экспоненциальное возрастание интенсивности $s$-компоненты аппроксимируется выражением

$$
I_{s-m}=I_{0} \cdot \exp \left(t / \tau_{i-m}\right),
$$

где $I_{0}-$ значение $I_{s-m}$ при $t \approx 0$, т.е. когда в GaAs, предположительно, доминирует еще спонтанное излучение. Обнаружилось, что характерное время $\tau_{i-m}$ экспоненциального возрастания интенсивности $s$-компоненты

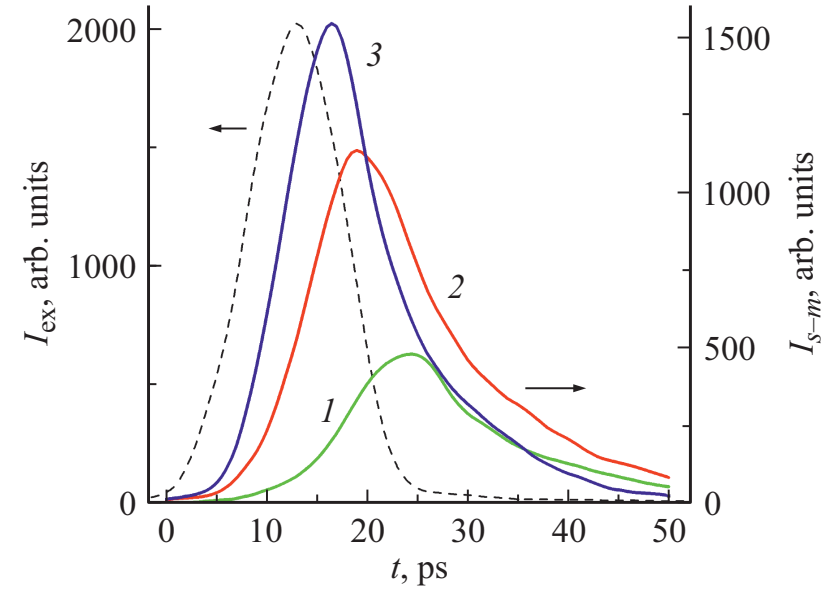

Рис. 1. Хронограммы импульсов накачки $I_{\mathrm{ex}}(t)$ и избранных спектральных компонент $s$-излучения $I_{s-m}(t)$ с энергиями фотона $\hbar \omega$, эВ: $1-1.387,2-1.396,3-1.406$.

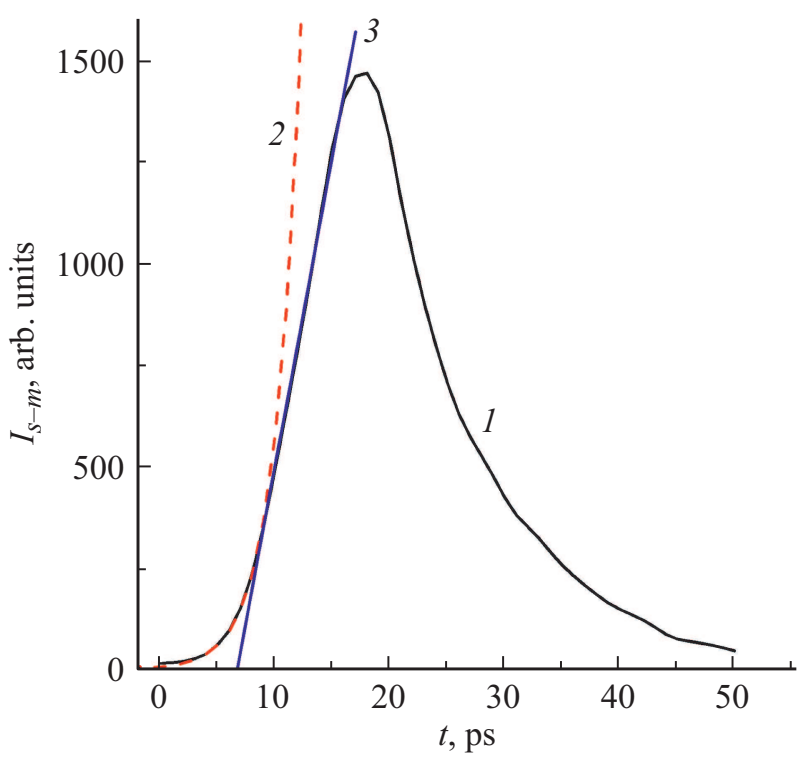

Рис. 2. Хронограмма $I_{s-m}(t)$ спектральной компоненты излучения с энергией фотона $\hbar \omega=1.403$ эВ (1). Кривые 2 и 3 поясняются в тексте.

в начале ее фронта увеличивалось при уменьшении энергии фотона компоненты (кривая 1 на рис. 3 ).

Второй график на рис. 3 показывает, как характерное время $\tau_{r-m}$ релаксации интенсивности $I_{s-m}$ при спаде $s$-компоненты менялось с $\hbar \omega$. Этот спектр получен в работе [1]. Время $\tau_{r-m}$ замедлялось из-за разогрева носителей заряда излучением. Поясним влияние этого разогрева. В работе [4] было получено аналитическое выражение характерного времени $\tau_{T}$ остывания оптически накачанных носителей заряда в GaAs. Остывание происходило в присутствии стимулированного излучения GaAs, которое создавало дополнительный нагрев носителей заряда, увеличивавший $\tau_{T}$. В работе [7] было показано, что благодаря взаимосвязи плотности и температуры неравновесных носителей заряда, возникающей в 


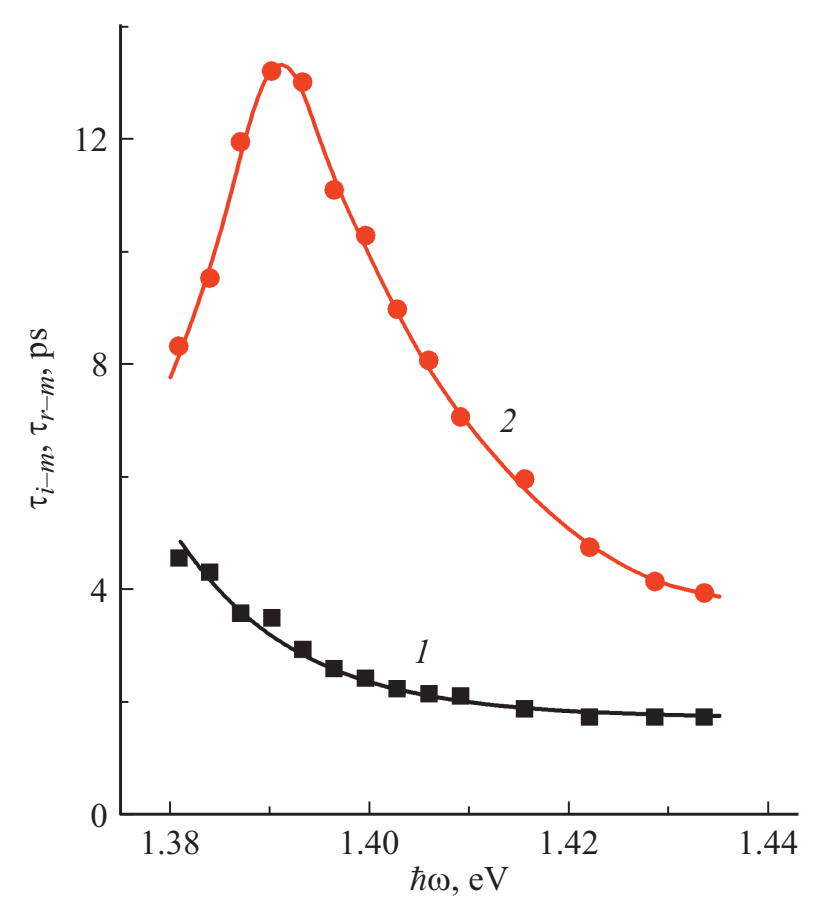

Рис. 3. Зависимости от энергии фотона $\hbar \omega$ характерного времени $\tau_{i-m}$ экспоненциального возрастания (1) и характерного времени $\tau_{r-m}$ релаксации (2) интенсивности спектральных компонент $s$-излучения. Сплошные линии проведены для наглядности.

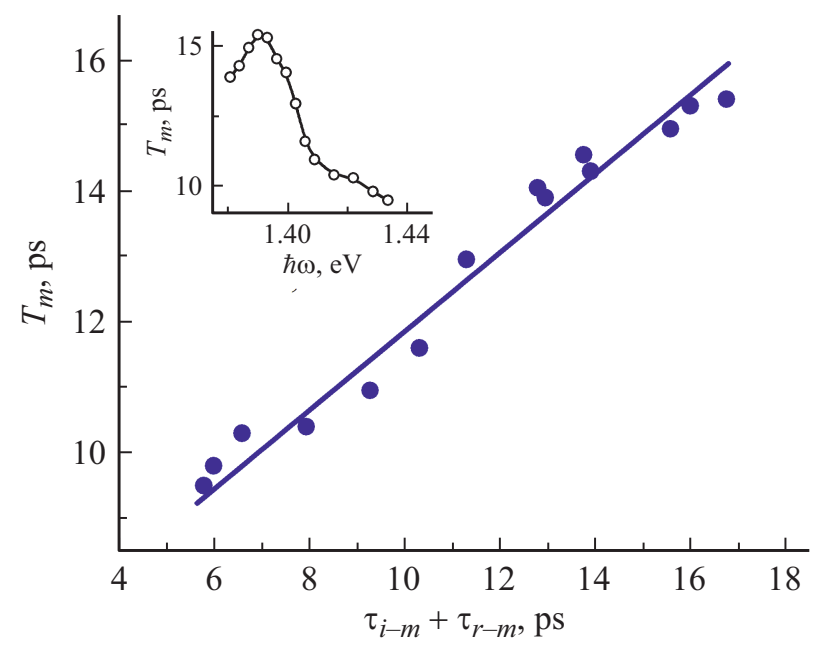

Рис. 4. Зависимость длительности $T_{m}$ спектральной компоненты $s$-излучения от суммы времен $\left(\tau_{i-m}+\tau_{r-m}\right)$, на вставке спектр этой длительности $T_{m}(\hbar \omega)$.

GaAs при интенсивном $s$-излучении, время $\tau_{T}$ и характерное время $\tau_{r}$ релаксации интенсивности $s$-излучения становятся взаимосвязаны. Отсюда следовало выражение для времени $\tau_{r}$, доступного для прямых измерений,

$$
\tau_{r} \approx(2 / 3) \tau_{T} \approx(2 / 3)\left(A+B \tau_{p} T_{c}^{1 / 2} E_{g}\right) \tau_{h},
$$

где $A=6.3, B=0.36, \tau_{h} \approx 0.8$ пс - время релаксации энергии электронно-дырочной плазмы (ЭДП) за счет эмиссии оптических фононов с учетом разогрева последних [8], $\tau_{r}, \tau_{p}, \tau_{h}$ измеряются в пс, $T_{c}$ - температура ЭДП в К, $E_{g}$ в эВ. Первое слагаемое в выражении (2) учитывает разогрев ЭДП, связанный с тем, что энергия носителей заряда, участвующих в стимулированной рекомбинации, меньше средней энергии носителей в ЭДП. Второе слагаемое учитывает разогрев ЭДП из-за внутризонного поглощения собственного излучения. Время $\tau_{p}$ — это характерное время движения фотона излучения в активной среде, пока фотон не покинет эту среду или не будет поглощен,

$$
\tau_{p}^{-1} \approx c \cdot \chi^{-1}\left(\beta+D^{-1}\right) .
$$

Здесь $\chi=3.6$ - показатель преломления $\mathrm{GaAs}$, $\beta=\sigma n-$ коэффициент внутризонного поглощения света, $\sigma=1.5 \cdot 10^{-17} \mathrm{~cm}^{2}[9], D-$ диаметр активной области. Следовавшая из (2) и (3) взаимосвязь времени $\tau_{r}$ и диаметра $D$ удовлетворительно согласовалась с измеренной в эксперименте [5] зависимостью $\tau_{r}(D)$, где $D$ относился к активной области интегрального по спектру $s$-излучения. К последней зависимости была близка и экспериментально полученная зависимость $\tau_{r-m}(L)$ в работе [10] в согласии с тем, что $L-$ это диаметр активной области $s$-компоненты.

В работе [1] был измерен спектр длительности на полувысоте (FWHM) $s$-компоненты $T_{m}(\hbar \omega)$ (см. вставку на рис. 4). Сопоставляя его со спектрами $\tau_{r-m}(\hbar \omega)$ и $\tau_{i-m}(\hbar \omega)$, обнаруживается приблизительно линейная зависимость

$$
T_{m} \approx 6.2+0.57\left(\tau_{i-m}+\tau_{r-m}\right) .
$$

\section{3. Линейное возрастание на фронте $s$-компоненты, его связь с предшествующим экспоненциальным возрастанием и с нагревом носителей $s$-излучением}

На основной части фронта интенсивность $s$-компоненты возрастала со временем линейно. Это иллюстрируется на рис. 2 касанием к фронту прямой 3 . Переход от экспоненциального к линейному усилению происходит так, что существует интервал времени, где экспоненциальное и линейное усиление перекрываются. Линейное возрастание аппроксимируется формулой

$$
I_{s-m}=I_{1}\left[1+\gamma_{L}\left(t-t_{1}\right)\right]
$$

где индекс 1 означает начало линейного участка фронта, a $\gamma_{L}$ будем называть коэффициент линейного усиления. Обнаруженный спектр $\gamma_{L}(\hbar \omega)$ представлен кривой 1 на рис. 5. Снизу к графику проведена пунктиром касательная, чтобы нагляднее показать, что, во-первых, два излома на графике при $\hbar \omega \approx 1.390$ и 1.422 эВ могут интерпретироваться как модуляция, возникающая из-за 
интерференции; во-вторых, нивелированный спектр может рассматриваться как квазилинейный. Возникновение интерференции в волноводной гетероструктуре объяснено в [11], а обнаружено еще раньше. Было установлено, что для гетероструктур, используемых в наших опытах, период интерференционной модуляции должен быть

$$
P \approx m \cdot \xi,
$$

где $m$ - целое число, $\xi \approx 10$ мэВ. В [11] $m=1$ соответствовало более мощному излучению, а $m=2$ более слабому. Теперь мы получили проявление модуляции с $m=3$, как свидетельствует интервал между изломами $P=32$ мэВ $\approx 3 \xi$ (рис. 5). Обнаруживается, как описано далее, что коэффициент линейного усиления на фронте связан с характерными временами экспоненциального возрастания в начале фронта и релаксации на спаде $s$-компоненты.

На рис. 6 кривой 1 представлен соответствующий спектру 1 на рис. 3 спектр коэффициента экспоненци-

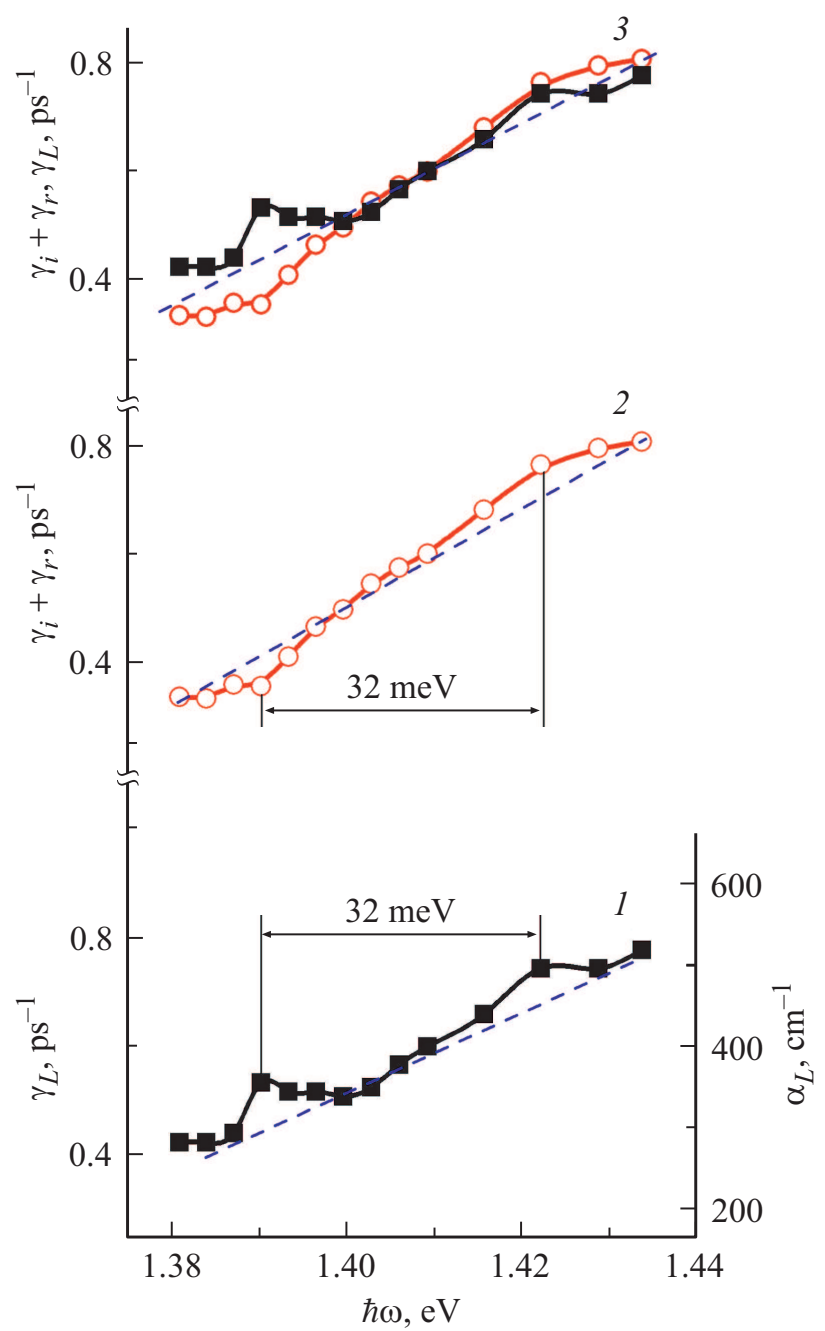

Рис. 5. Спектры коэффициента линейного усиления $\gamma_{L}(\hbar \omega)(1)$ и суммы $\left(\gamma_{i}(\hbar \omega)+\gamma_{r}(\hbar \omega)\right)$ (2) коэффициентов усиления, экспоненциального и обусловленного ЭТН. Пунктирные линии проведены для наглядности. Пояснение правой оси ординат см. в тексте.

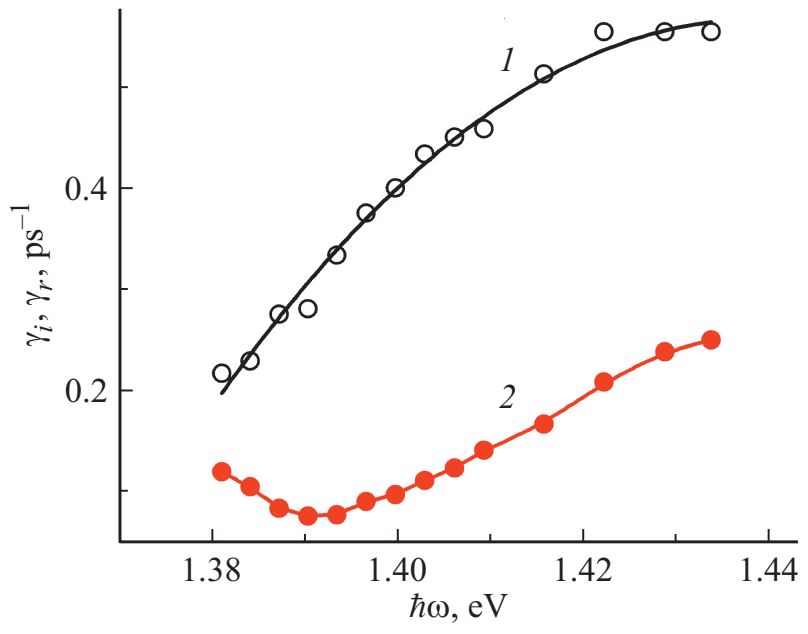

Рис. 6. Спектр коэффициента экспоненциального усиления $\gamma_{i}(\hbar \omega)(1)$. Спектр коэффициента усиления $\gamma_{r}(\hbar \omega)(2)$, обусловленного ЭТН. Сплошные линии проведены для наглядности.

ального усиления в начале фронта $\gamma_{i}=1 / \tau_{i-m}=f(\hbar \omega)$. Экспериментальные точки аппроксимируются гладкой кривой, являющейся графиком зависимости

$$
\gamma_{i}=k\left(\hbar \omega-E_{g}\right)^{1 / 2} / \hbar \omega,
$$

где $k=\mathrm{const}, E_{g}=1.376$ эВ - величина, следующая из подгонки кривой к точкам и представляющаяся реалистичной в качестве ширины перенормированной запрещенной зоны. Выражение (7) соответствует хорошо известной зависимости от $\hbar \omega$ и $E_{g}$ коэффициента усиления „слабого“ света.

Обозначим ЭТН транспорт неравновесных носителей в энергетическом пространстве на уровни в области экстремумов зон проводимости и валентной, откуда они рекомбинируют. Можно предположить, что скорость ЭТН на конкретный энергетический уровень обратно пропорциональна характерному времени релаксации на этот уровень остывающих носителей, а значит, учитывая левое равенство в (2), обратно пропорциональна и $\tau_{r-m}$. Допустим, что вклад ЭТН в усиление на фронте $s$-компоненты характеризуется коэффициентом усиления

$$
\gamma_{r}=1 / \tau_{r-m}
$$

Спектр $\gamma_{r}(\hbar \omega)$ представлен на рис. 6 кривой 2. Обнаруживается, что сумма спектров коэффициентов усиления, экспоненциального и обусловленного ЭТН, представленная кривой 2 на рис. 5, приблизительно равна спектру линейного усиления, т. е.

$$
\gamma_{i}(\hbar \omega)+\gamma_{r}(\hbar \omega) \approx \gamma_{L}(\hbar \omega)
$$

Это приближенное равенство иллюстрируют кривые 3 на рис. 5. Отклонение от строгого равенства создается, во-первых, обсуждавшейся выше модуляцией спектра 
$\gamma_{L}(\hbar \omega)$. Во-вторых, обнаруживающейся модуляцией суммарного спектра $\gamma_{i}(\hbar \omega)+\gamma_{r}(\hbar \omega)$. Для того чтобы представить ее нагляднее через график спектра, представленного отдельно кривой 2 на рис. 5, проведена пунктирная прямая. Как видно на рис. 5 , при $\hbar \omega \approx 1.422$ эВ локальные максимумы модуляции спектров $\gamma_{i}(\hbar \omega)+\gamma_{r}(\hbar \omega)$ и $\gamma_{L}(\hbar \omega)$ совпадают, а при $\hbar \omega \approx 1.390$ эВ локальному минимуму модуляции суммарного спектра $\gamma_{i}(\hbar \omega)+\gamma_{r}(\hbar \omega)$ соответствует локальный максимум модуляции спектра $\gamma_{L}(\hbar \omega)$, т.е. периоды сравниваемых двух модуляций отличаются вдвое. Модуляция спектра $\gamma_{i}(\hbar \omega)+\gamma_{r}(\hbar \omega)$ во многом возникает благодаря немонотонности спектра $\gamma_{r}(\hbar \omega)$, которая, по-видимому, корректируется условием (6). На этом этапе можно заключить, что равенство (9) приближенно выполняется, с погрешностью, отображаемой отклонениями кривых 3 от проведенного через них пунктира на рис. 5.

\section{4. Обсуждение экспериментальных результатов}

Экспериментально установлено, что интенсивность $s$-компоненты растет со временем: (а) в начале фронта, пока она относительно мала, экспоненциально (аналогично закону Бугера для слабой бегущей волны); (б) на основной части фронта, где интенсивность уже большая, - линейно. Релаксирует $s$-компонента, как было установлено ранее, с характерным временем, пропорциональным характерному времени остывания ЭДП и немного меньшим его. Смена экспоненциального усиления линейным - это нелинейное оптическое явление. Оно было теоретически выведено, например, в [12,13], но так, что у нас нет возможности сопоставить теоретические выводы с экспериментальными результатами.

Обнаружилась неизменность сначала коэффициента экспоненциального усиления во времени $\gamma_{i}$, а следом коэффициента линейного усиления $\gamma_{L}$, в рамках какого-то одного, экспоненциального или линейного, режима, при непрерывно растущей накачке. Это внешне подобно ситуации, рассмотренной в [14] для четырехуровневого лазера. Там, если скорость накачки превысит пороговое значение, при котором начинается вынужденная генерация фотонов, то инверсия приобретает критическое значение и будет оставаться неизменной, даже если стационарную накачку станут еще увеличивать. Но нельзя автоматически распространять вывод, полученный для четырехуровневого лазера в стационарном режиме, на фотонакачанный полупроводник, генерирующий интенсивное излучение в динамическом режиме.

Определены спектры коэффициентов усиления экспоненциального $\gamma_{i}$ и линейного $\gamma_{L}$. Изменение $\gamma_{i}$ в зависимости от превышения энергии фотона над шириной запрещенной зоны соответствует общеизвестному корневому закону (7), выведенному для поглощения и усиления слабого света $[9,15]$. Спектр коэффициента $\gamma_{L}$ имеет квазилинейную форму, напоминающую образовывавшуюся в [6] при выжигании провала в спектре усиления. Таким образом, квазилинейная форма спектра, сменяющая корневую при наступлении режима линейного усиления, соответствует, по-видимому, еще и „выжиганию провала“ в спектре усиления.

Определение коэффициента линейного усиления выражением (9) было по сути получено эмпирически. Оно прояснило, каким должен быть коэффициент в этом режиме, возникающем при наступлении этапа насыщения усиления, когда на усиление начинает влиять интенсивность излучения. В (9) присутствует член $\gamma_{r}$, определяющий влияние ЭТН, замедленного из-за нагрева носителей излучением, на линейное усиление. Тем самым доказывается, что нагрев излучением тормозит не только релаксацию, но и возрастание $s$-компоненты на фронте. Благодаря нагреву формируется ООС [4]: возрастание интенсивности излучения приводит к усилению нагрева носителей за счет внутризонного поглощения, из-за чего уменьшается инверсия заселенности и, как следствие, ослабляется излучение. Эта ООС может, в частности, способствовать стабилизации коэффициента линейного усиления.

Длительность $s$-компоненты (FWHM) $T_{m}$ оказывается приблизительно пропорциональной характерным временам $\tau_{i-m}$ и $\tau_{r-m}$. По-видимому, соответствующие им процессы, во-первых, экспоненциального усиления интенсивности $s$-компоненты, аналогично закону Бугера, во-вторых, релаксации накачанных носителей, замедленной из-за их разогрева излучением, в основном и определяют $T_{m}$. Получая формулу для $T_{m}$, мы для наглядности, чтобы было ближе к физическому смыслу, оперировали временами, а формулу для $\gamma_{L}-$ коэффициентами усиления. Если же использовать только устоявшиеся со временем термины (коэффициент усиления слабого света, время релаксации, замедленной из-за нагрева носителей излучением), то (4) и (9) будут представлены в виде

$$
\begin{gathered}
T_{m} \approx 6.2+0.57\left(1 / \gamma_{i}+\tau_{r-m}\right), \\
\gamma_{L}(\hbar \omega) \approx \gamma_{i}(\hbar \omega)+1 / \tau_{r-m}(\hbar \omega),
\end{gathered}
$$

в котором еще и наглядна антикорреляция $T_{m}$ и $\gamma_{L}$.

Определявшиеся выше коэффициенты $\gamma_{i}, \gamma_{r}, \gamma_{L}$ относятся к усилению со временем. Обычно в теории определяются коэффициенты усиления $\alpha$ в пространстве, но для бегущей волны. В активной среде волноводной гетероструктуры усиливаются парциальные волны, движущиеся навстречу друг другу и формирующие почти стоячую волну, благодаря которой образуется брэгговская решетка заселенности [11]. Нам не известны работы, где бы была аналитически получена связь $\gamma$ и $\alpha$. Если же сопоставить спектр $\gamma_{L}(\hbar \omega)$ с измеренным методом „рump-probe“ спектром коэффициента усиления $\alpha(\hbar \omega)$, представленным на рис. 2 в работе [6], то оказывается, что в диапазоне $\hbar \omega=1.381-1.414$ эВ, где они перекрываются, эти спектры приближенно связаны соотношением

$$
\alpha \approx 2 \gamma \chi / c R
$$

где $R \approx 0.36$ - оценка коэффициента отражения брэгговской решеткой [11], а коэффициент 2, возможно, 
связан с тем, что парциальные волны усиливаются только в местоположении пучностей стоячей волны. Эмпирическое соотношение (12) представляется логичным уже в том, что для фиксированного усиления со временем $\gamma=$ const требуется тем меньший $\alpha$, чем сильнее положительная обратная связь, создаваемая брэгговской решеткой, т.е. чем больше $R$. Тем не менее сопоставлялись спектры, измеренные в разных экспериментах и расположенные в целом в несколько отличающихся спектральных интервалах, поэтому соотношение (12) можно воспринимать как кажущееся реалистичным, но нуждающееся в специальном изучении и корректировке. Это отдельный вопрос на будущее. Получаемые для спектра $\gamma_{L}(\hbar \omega)$ из соотношения (12) значения $\alpha$ относятся к правой оси ординат на рис. 5 и приводятся только для того, чтобы представить порядок величины $\alpha$, который с учетом сделанных выше оговорок, можно надеяться, отображает указанное соотношение.

\section{5. Заключение}

Показано, что в начале фронта $s$-компоненты коэффициент усиления корневым образом зависит от разности $\hbar \omega-E_{g}$, и усиление согласуется с законом Бугера. А именно реализуются известные представления для режима „слабого“ света. На фронте по мере возрастания интенсивности $s$-компоненты возникал режим линейного усиления, предполагавшийся теоретически и теперь экспериментально реализованный. Это - нелинейное оптическое явление. Установлен коэффициент линейного усиления, получаемый, как оказалось, добавлением к коэффициенту экспоненциального усиления, определенному для „слабого“ света, еще и коэффициента усиления, определяемого энергетическим транспортом носителей к экстремумам зон проводимости и валентной (ЭТН). Поскольку ЭТН замедлен из-за нагрева носителей излучением, то тем самым выявляется замедление возрастания излучения на фронте, вызванное таким нагревом. Влияние нагрева на релаксацию излучения было экспериментально доказано в предыдущих работах, пользуясь теоретическим определением времени остывания носителей в работе [4]. Влияние нагрева на фронт в доступной для экспериментальной проверки форме в той теоретической работе мы не нашли. Но теперь, как сказано выше, оно выявлено экспериментально. Обнаруживается, что при наступлении режима линейного усиления меняется не только коэффициент усиления, но и форма спектра. Вместо корневого спектра при экспоненциальном усилении возникает квазилинейный спектр (в доступном для чувствительности нашей измерительной аппаратуры спектральном диапазоне). Экспериментально показано, что в каждом из режимов усиления (экспоненциального и линейного) соответствующие им коэффициенты усиления, отличные один от другого, оставались неизменными. Длительность $s$-компоненты оказалась пропорциональна опять же двум параметрам режимов экспоненциального усиления и релаксации: сумме характерных времен первого и второго.
Представленная выше работа пополняет экспериментальную информацию о динамике спектральных компонент интенсивного стимулированного излучения, генерируемого в полупроводниковой гетероструктуре. Особенно это относится к мало исследованной динамике в состоянии насыщения усиления. Пополнилось представление о значительной роли разогрева носителей собственным излучением полупроводника. Установленные эмпирические зависимости дадут вклад в экспериментальную базу для развития теории указанной динамики. Они должны быть полезны и при анализе функционирования лазерных усилителей, а также полупроводниковых лазеров и суперлюминесцентных диодов, предназначаемых для генерации мощных сверхкоротких световых импульсов.

\section{Финансирование работы}

Работа выполнена в рамках государственного задания.

\section{Конфликт интересов}

Авторы заявляют, что у них нет конфликта интересов.

\section{Список литературы}

[1] Н.Н. Агеева, И.Л. Броневой, Д.Н. Забегаев, А.Н. Кривоносов. ЖЭТФ, 143, 634 (2013).

[2] Н.Н. Агеева, И.Л. Броневой, Д.Н. Забегаев, А.Н. Кривоносов. ЖЭТФ, 144, 227 (2013).

[3] Н.Н. Агеева, И.Л. Броневой, Д.Н. Забегаев, А.Н. Кривоносов. ФТП, 55, 113 (2021).

[4] Ю.Д. Калафати, В.А. Кокин. ЖЭТФ, 99, 1793 (1991).

[5] Н.Н. Агеева, И.Л. Броневой, Д.Н. Забегаев, А.Н. Кривоносов. ФТП, 53, 1471 (2019).

[6] Н.Н. Агеева, И.Л. Броневой, А.Н. Кривоносов, С.Е. Кумеков, С.В. Стеганцов. ФТП, 36, 144 (2002).

[7] Н.Н. Агеева, И.Л. Броневой, А.Н. Кривоносов. ФТП, 35, 65 (2001).

[8] С.Е. Кумеков, В.И. Перель. ЖЭТФ, 94, 346 (1988).

[9] J.S. Blakemore. J. Appl. Phys., 53, R123 (1982).

[10] Н.Н. Агеева, И.Л. Броневой, Д.Н. Забегаев, А.Н. Кривоносов. ФТП, 55, 121 (2021).

[11] Н.Н. Агеева, И.Л. Броневой, Д.Н. Забегаев, А.Н. Кривоносов. ФТП, 54, 1018 (2020).

[12] L.W. Casperson. J. Appl. Phys., 48, 256, (1977).

[13] В.Д. Соловьев. Физика лазеров. Текст лекций (4-й курс). http://elib.spbstu.ru/d1/2313.pdf/download/2313.pdf

[14] О. Звелто. Принцииь лазеров (СПб., Лань, 2008).

[15] В.Л. Бонч-Бруевич, С.Г. Калашников. Физика полупроводников (М., Наука, 1990).

Редактор Г.А. Оганесян 


\section{Influence of heating of charge carriers by intrinsic stimulated picosecond emission in GaAs on the linear increase at the front and the duration of the spectral component of this emission}

N.N. Ageeva, I.L. Bronevoi, D.N. Zabegaev, A.N. Krivonosov

Kotel'nikov Institute of Radioengeneering and Electronics, Russian Academy of Sciences, 125009 Moscow, Russia

Abstract During high-power optical picosecond pumping of a thin GaAs layer, which is part of the $\mathrm{Al}_{x} \mathrm{Ga}_{1-x} \mathrm{As}-\mathrm{GaAs}-\mathrm{Al}_{x} \mathrm{Ga}_{1-x} \mathrm{As}$ heterostructure, intense stimulated picosecond emission arises in it. When analyzing the pulses of spectral components of the emission measured in real time, the coefficients of exponential and then linear amplification of the components at the front were determined. In this case, the influence of the heating of charge carriers by emission on the front of the components was found. The dependence of the component duration (FWHM) on the characteristic times of rise at the front and of relaxation at the decay (also slowed down by emission heating of carriers) of the component is obtained. 